Review Article

The New (2018) European

Hypertension Guidelines an overview

\section{\& comments}

\author{
Mohsen Ibrahim $\mathrm{M}^{*}$ \\ President, Egyptian Hypertension Society, Professor of Cardiology, Cairo University, Egypt
}

The European Society of Cardiology (ESC) and the European Society of Hypertension (ESH) jointly developed a series of hypertension guidelines in the years 2003, 207 and 2013. The most recent guidelines were issued by the two societies in August this year (2018) and were published in the European Heart Journal. The new guidelines are printed in more than 90 pages and cover almost all aspects of hypertension based on extensive review of literature giving highest priority to data from randomized controlled trials and well conducted meta-analysis. In important areas where there is inadequate or no evidence, guidelines authors resort to expert opinion. The text was developed over approximately 24 months and was reviewed by representatives of ESC and ESH national hypertension societies. Although it is less than five years since the last hypertension European guidelines in 2013, the recent 2018 guidelines show important differences in diagnosis and treatment strategies with the addition of new sections and recommendations on management of hypertensive emergencies, hypertension in women and pregnancy, different ethnic groups, chronic obstructive pulmonary disease, cancer therapies, peri-operative management, sexual dysfunction and perioperative management.

In this message, I will give an overview of the main recommendations in the European guidelines under two headings: diagnosis and treatment and I will conclude by comments.

\section{An Overview}

\section{Diagnosis}

The 2018 guidelines [1] maintain the same definition and classification of hypertension as in 2013 guidelines. Hypertension is defined as an office SBP $\geq 140 \mathrm{mmHg}$ and/ or DBP $\geq 90 \mathrm{mmHg}$; which is equivalent to a 24-hours average AMBPM of $\geq 130 / 80 \mathrm{mmHg}$ or home blood pressure measurements (HBPM) average $\geq 135 / 85 \mathrm{mmHg}$.

Out of office blood pressure measurements (ABPM and HBPM) are recommended to confirm the diagnosis

\section{More Information}

*Address for Correspondence: Mohsen Ibrahim M, President, Egyptian Hypertension Society, Professor of Cardiology, Cairo University, Egypt, Tel: 00201222273816; Email: ehs@link.net; mibrahim_02@yahoo.com

Submitted: 12 November 2018

Approved: 23 July 2019

Published: 24 July 2019

How to cite this article: Mohsen Ibrahim M. The New (2018) European Hypertension Guidelines an overview \& comments. Ann Clin Hypertens. 2019; 3: 039-042.

DOI: 10.29328/journal.ach.1001017

Copyright: (c) 2019 Mohsen Ibrahim M. This is an open access article distributed under the Creative Commons Attribution License, which permits unrestricted use, distribution, and reproduction in any medium, provided the original work is properly cited

Check for updates

of hypertension as an alternative to repeated office visits. Automated multiple blood pressure readings with the patient seated alone give blood pressure values lower than those obtained by conventional office blood pressure measurements and are similar even less than daytime ABPM and HBPM and help to rule out white-coat hypertension.

- Blood pressure must be measured in the two arms during the first visit, a consistent and significant differences in SBP between the two arms ( $>15 \mathrm{mmHg}$ ) is associated with increased cardiovascular (CV) risk.

- White-coat hypertension does not appear to be clinically innocent.

- It is recommended to repeat blood pressure measurements at least every 5 years for those with optimal blood pressure $(<120 / 80 \mathrm{mmHg}$ ) and every 3 years for those with normal blood pressure (120-129/80-89 $\mathrm{mmHg}$ ) and annually for high normal blood pressure (130-139/85-89 mmHg).

- Home or ABP monitoring is indicated when white coat or masked hypertension are suspected, in resistant hypertension, when there is considerable variability in office blood pressure or symptoms of hypotension during treatment.

- CV risk assessment with the SCORE system* is recommended for hypertensive patients who are not already at high or very high risk due to established CV disease, renal disease or diabetes or a markedly elevated single risk factor or hypertensive LV hypertrophy. 
- Increased serum uric acid is independently associated with increased CV risk.

- Hypertension and cancer therapy: because of the pressor effect of widely used anticancer drugs, office blood pressure should be measured weekly during the initial part of the first cycle of therapy and at least every 2-3 weeks thereafter.

- SBP appears to be a better predictor of events than DBP after the age of 50 years.

- Albumin: creatinine ratio (ACR) measured from early morning spot urine sample should be documented in all hypertensive patients.

\section{Treatment}

The new guidelines were less conservative in treatment thresholds in contrast to 2013 guidelines. The recommended blood pressure treatment target is blood pressure $<140 / 90$ $\mathrm{mmHg}$ in all patients and to $130 / 80 \mathrm{mmHg}$ or lower in most patients if tolerated. In patients $<65$ years, it is recommended that SBP should be lowered to a blood pressure range of 120 $129 \mathrm{mmHg}$ in most patients.

- A more aggressive approach regarding blood pressure lowering in elderly patients ( $>65$ years and over 80 years). SBP should be targeted to a blood pressure range of 130-139 $\mathrm{mmHg}$ which is lower than previous guidelines.

- DBP target $<80 \mathrm{mmHg}$ should be considered for all hypertensive patients independent of the level of risk and co-morbidities.

- It is recommended to lower blood pressure to a range of 130-139 mmHg in patients with chronic kidney disease and to $130 \mathrm{mmHg}$ and lower if tolerated.

*Score system estimates the 10 -year risk of a first fatal atherosclerotic event, in relation to age, sex, smoking, total cholesterol level and SBP.

- Drug treatment for high normal blood pressure (130$139 / 85-89 \mathrm{mmHg}$ ) when CV risk is very high due to established cardiovascular disease and in patients with grade 1 hypertension (140-159/90-99 $\mathrm{mmHg}$ ) at low or moderate risk without evidence of target organ damage if the patient remains hypertensive after a period of lifestyle of 3-6 months.

- The 2018 guidelines [1] recommended to initiate antihypertensive treatment with a two drug combination preferably in a single pill (SPC) except in frail older patients and those at low risk and with grade 1 hypertension.

- A simplified treatment algorithm was recommended with the preferred use of an ACE inhibitor or ARB combined with CCB and/or thiazide-like diuretic for most patients, with beta-blockers used for specific indications.

- Use of device-based therapy is not recommended for the routine treatment of hypertension.

- Major uncertainties remain to the clinical role of renal denervation outside of clinical studies, which should be performed in carefully selected patients, at specialized hypertension centers and by experienced operators.

- In hypertensive patients with an acute cerebrovascular event, antihypertensive treatment is recommended immediately for transient ischemic attack and after several days in ischemic stroke.

- New blood pressure lowering drugs for resistant hypertension: nitric oxide donors, vasopressin antagonists, neural endopeptidose inhibitors and endothelin antagonists, are under investigation.

- Presence of COPD has an impact on the selection of antihypertensive drugs. Diuretics are not recommended for widespread use in hypertensive patients with COPD while the use of cardiac beta-selective beta blockers is safe.

- There is a key role of nurses and pharmacists in long term management of hypertension.

\section{Comments on the new (2018) European Hypertension Guidelines}

- The new European guidelines [1] recommend a more aggressive approach in lowering blood pressure and the choice of lower blood pressure targets in the majority of patients. This was based upon results from recent clinical trials, systemic review of literature and meta-analysis which suggest that blood pressure lowering to levels below those recommended in current guidelines (SBP of less than $140 \mathrm{mmHg}$ ) will reduce the risk of cardiovascular events [2-4]. This approach though scientifically sound is difficult to apply in many countries.

- A more stringent blood pressure targets (on treatment blood pressure values of $\leq 130 / 80 \mathrm{mmHg}$ in the general population) will make the achievement of blood pressure control more challenging and more expensive.

- Offering pharmacologic treatment to low risk patients with mild hypertension who constitute a good proportion of our patient's population is unrealistic. Priority of drug therapy should be given to patients at high cardiovascular risk; namely established cardiovascular disease, diabetes, chronic kidney disease or multiple atherosclerotic cardiovascular risk factors. 
- Drug treatment based on blood pressure alone is likely to have little individual effect in low risk patients with mild hypertension. Levels at which SBP is related to increased mortality vary with age and sex.

- No outcome trial has ever tested or proven the benefits of drug treatment in younger patients (18-59 years old) or mild hypertension (BP $<160 / 100 \mathrm{mmHg}$ ) with a low estimated 10-year ASCVD risk. A substantial proportion of the population with $\mathrm{SBP} \geq 140 \mathrm{mmHg}$ is at no increased risk.

- The feasibility and risk benefit ratio of decades of drug treatment in young lower-risk people is not known. Adverse events (e.g. hypotension, syncope, azotemia, electrolyte disorders, allergy) may be more than reduction in atherosclerotic cardiovascular disease events.

- A recent review by Sundstorm J, et al. [5] showed that antihypertensive therapy in patients with mild hypertension and no previous history of CV disease, does appear to reduce the risk of overall death, CV death and stroke, but not cardiovascular events, coronary events and heart failure.

- In young (18-55 years old)and low risk patients (< 5-7.5\%/10 years) with a blood pressure $<160 / 100$ $\mathrm{mmHg}$ a trial of a period of lifestyle therapy (3-12 months) prior to starting medications may be a wise policy.

- Initiating treatment with combination therapy (single pill combination) for all patients may be a good policy, but what about patients who may respond to single drug i.e. why give two drugs if one drug can do the job? This recommendation is almost unique for the European guidelines. Other international guidelines recommend to initiate drug therapy for stage 1 hypertension when indicated by one of the four or five standard groups or a single pill combination.

- Guidelines made no reference to the possible role of sugar intake, excessive air and noise pollution as emerging risk factors for hypertension which might need interventions.

- These guidelines are not practical for developing countries [6]. Simply we cannot afford to treat everyone with a blood pressure $\geq 140 / 90 \mathrm{mmHg}$, taking into consideration the indiscriminate diagnosis of hypertension based on a single blood pressure reading or single rapid office visit and without taking the necessary precautions to obtain accurate measurements in a busy outpatient clinic where half the patients might have white-coat hypertension.

- Several studies have shown that routine manual blood pressure reading (SBP/DBP) are on average $9 / 6 \mathrm{mmHg}$ higher compared with the corresponding research quality manual blood pressure measurements.

- National or regional hypertension guidelines are needed when the European guidelines are not practical and difficult to follow. We have to realize that there are variations in lifestyle, ethnic variations in response to drug therapies and equally important are resource availability. What is reasonable to recommend in wealthy country will be impossible in many poor resource environments.

- I personally find the Canadian Hypertension Guidelines (CHEP) $[7,8]$, which are updated every year more suitable for our patients and should be considered as an important reference when we plan to update our recent Egyptian guidelines [9].

- The CHEP guidelines take a more conservative approach regarding diagnosis and treatment of hypertension, e.g. if systolic blood pressure is ranging $140-160 \mathrm{mmHg}$ and diastolic blood pressure is $90-100 \mathrm{mmHg}$ you have to repeat office measurements on 4 to 5 separate occasions or use home or ambulatory blood pressure monitoring before diagnosis is made. At office visit 4 or 5 average blood pressure across all visits $\geq 140 / 90$ mmHg. Antihypertensive therapy should be prescribed for average diastolic blood pressure measurements $\geq 100 \mathrm{mmHg}$ or average systolic blood pressure $\geq$ $160 \mathrm{mmHg}$ in patients without target organ damage (TOD) or other cardiovascular risk factors, and should be strongly considered for average diastolic blood pressure readings $\geq 90 \mathrm{mmHg}$ or for average systolic blood pressure $\geq 140 \mathrm{mmHg}$ in presence of TOD or other cardiovascular risk factors.

- Initial therapy should be either monotherapy or single pill combination. Additional antihypertensive therapy if target blood pressure levels are not achieved with standard dose monotherapy.

The following points need to be clarified:

- All drug classes are similar in their ability to reduce the risks of cardiovascular events, even in the elderly or diabetics?

- Cardiovascular protection depends on blood pressure lowering per se, regardless of how it is achieved.

- ARBs may not reduce the risk of MI.

- Role of diuretic / B-blocker combinations in reducing the risks of stroke and MI in the elderly.

- Metabolic disturbances associated with diuretic/bblocker combinations can be avoided by the use of potassium sparing diuretic therapy combined with highly B-1 selective beta blockade. 
- Finally, guidelines are intended to provide a framework but should not replace clinical judgment.

\section{References}

1. Bryan W, Giuseppe M, Wilko S, Enrico A, Michel Azizi Michel Burnier, Denis L Clement, et al. 2018 ESC/ESH Guidelines for the management of arterial hypertension. European Heart Journal. 2018; 39: 3021-3104.

2. Ettehad D, Emdin CA, Kiran A, Anderson SG, Callender T, et al. Blood pressure lowering for prevention of cardiovascular disease and death: a systematic review and meta-analysis. Lancet. 2016; 387: 957-967. PubMed: https://www.ncbi.nlm.nih.gov/pubmed/26724178

3. Thomopoulos C, Parati G, Zanchetti A. Effects of blood pressurelowering on outcome incidence in hypertension: 5. Head-to-head comparisons of various classes of antihypertensive drugs - overview and meta-analyses. J Hypertens. 2015; 33: 1321-1341. PubMed: https://www.ncbi.nlm.nih.gov/pubmed/26039526

4. The SPRINT Research Group. A Randomized Trial of Intensive versus Standard Blood-Pressure Control. N Engl J Med. 2015; 373: 2103-2116.

5. Sundström J, Arima H, Jackson R, Turnbull F, Rahimi K, et al. Blood
Pressure Lowering Treatment Trialists' Collaboration. Effects of blood pressure reduction in mild hypertension: a systematic review and meta-analysis. Ann Intern Med. 2015; 162: 184-191. PubMed: https:// www.ncbi.nlm.nih.gov/pubmed/25531552

6. Ibrahim MM. Hypertension in Developing Countries: A major challenge for the future. Curr Hypertens Rep. 2018; 20: 38. PubMed: https:// www.ncbi.nlm.nih.gov/pubmed/29717393

7. Daskalopoulou SS, Dasgupta K, McBrien K, Butalia S, Zarnke KB, et al. Hypertension Canada's 2017 Guidelines for Diagnosis, Risk Assessment, Prevention, and Treatment of Hypertension in Adults. Can J Cardiol. 2017; 33: 557-576. PubMed: https://www.ncbi.nlm.nih. gov/pubmed/28449828

8. Nerenberg KA, Zarnke $K B$, Leung AA, Dasgupta $K$, Butalia $S$, et al. Hypertension Canada's 2018 guidelines for diagnosis, risk assessment, prevention, and treatment of hypertension in adults and children. Can J Cardiol. 2018; 34: 506-525. PubMed: https://www.ncbi.nlm.nih.gov/ pubmed/29731013

9. Ibrahim, M.M. The Egyptian hypertension society: Egyptian hypertension guidelines. Egyptian Heart Journal. 2014; 66: 79-132. 